\title{
Integrated Navigation for Flyback Reusable Booster Vehicles
}

\author{
By Pengxin HaN, Rongjun MU and Naigang CUI \\ Department of Aeronautics and Engineering, Harbin Institute of Technology, Harbin, China
}

(Received April 20th, 2010)

\begin{abstract}
Taking the engineering application of reusable booster vehicles (RBVs) as the research background, an effective navigation system scheme for rocket-powered auto-flyback RBVs is put forward in detail. First of all, the research state of RBVs in main research departments and three kinds of typically used feasible flyback options for RBVs are introduced. Based on an analysis of the characteristics of application background and engineering requirements, a feasible navigation scheme for a rocket-powered auto-flyback RBV is put forward, which consists of a strapdown inertial navigation system (SINS), global navigation satellite system (GNSS) and radio navigation equipment (radio). In order to realize navigation with high accuracy and dynamic adaptability, an improved dynamic information integration strategy is used for the navigation system, and simulation of the scheme based on a special trajectory is carried out. The theoretical analysis and simulation results indicate the effectiveness and feasibility of the navigation technologies.
\end{abstract}

Key Words: Flyback, Navigation, Information Fusion

\section{Nomenclature}

$X$ : state vector

$\beta$ : information-sharing coefficient

$P$ : estimate error covariance matrices

Subscripts

$i$ : number of sensors or filters

$g$ : global

\section{Introduction}

\subsection{Introduction to reusable booster vehicles}

The research and tests over the past few decades have indicated that a fully reusable signal-stage-to-orbit launch vehicle is impossible based on technologies applied today and in the near future. Therefore, multistage and partially reusable launch vehicles become the only effective choice to reduce space launch costs. In addition, these kinds of vehicles are mainly based on current technologies. ${ }^{1)}$

The reusable booster vehicle (RBV), which has evolved from the expendable booster and can be reused many times, is one of the most important directions for reusable transportation technologies in the near future. $\left.{ }^{2,3}\right)$ The development and application of RBVs could effectively promote progress on the relative technologies of reusable launch systems.

Until now, the main projects associated with RBVs include an advance space transportation system and small-size partially reusable launch vehicle of JAXA, ${ }^{4,5)}$ a Spaceplane in the U.S.A, ${ }^{3)}$ and Hopper and several two-stage-to-orbit programs of the ESA. ${ }^{1,2)}$ All of the launch vehicle concepts of these programs consist of RBVs and an expendable upper-stage. These types of transportation systems are partially reusable, and they are technically not too challenging and seem suitable to be targets for research activities in the next decade. ${ }^{\text {) }}$

(C) 2011 The Japan Society for Aeronautical and Space Sciences
After separation, RBV needs to flyback to the launch site or special landing area. In the projects mentioned above, the $\mathrm{RBV}$ is launched vertically and lands on a normal runway horizontally, such as the concepts presented by the ESA ${ }^{2,7)}$ and JAXA ${ }^{4)}$ in Fig. 1 and Fig. 2 respectively.

\subsection{Flyback options for RBV}

Until now, the first three most famous and mature options for flyback RBVs are unpowered glideback, ${ }^{8)}$ aero-powered flyback $^{9)}$ and rocket-powered flyback. ${ }^{5)}$ In the unpowered glideback option, as shown in Fig. 3, the separation velocity is low and the longitudinal range is limited. As the size of the RBV is small, before separation the increment to the launcher's total velocity caused by the RBV is small and limited as well.

As shown in Fig. 4, a RBV could fly back to the launch site or land at a secondary landing area with the aero-powered flyback option. Because RBV has several powered fly segments, maneuvering ability can obviously be improved. But secondary landing sites have to be selected and precautionary measures for a powered return flight must be included for the RBV. Simultaneously, additional aero-powered system may increase the total dry weight of the RBV.

In the rocket-powered flyback option, as shown in Fig. 5, the RBV flies a pitch-over attitude maneuver and a re-ignition portion. After turning around to the direction of the launch site, the RBV cuts off the rocket engines and starts reentry, followed by gliding back and then an auto landing. Generally, RBVs have a velocity of Mach 4.0-6.0 at the separation point, the maximum flight altitude is approximately $80-120 \mathrm{~km}$ and the flyback period is around $500-1,800 \mathrm{~s}$ from separation to landing.

\subsection{Navigation requirements}

When the RBV is flying back, the flight state is hypersonic and highly dynamic, with obvious and tremendous changes in aerodynamic force and aero load. In order to attain proper reentry states and realize an accurate automatic 


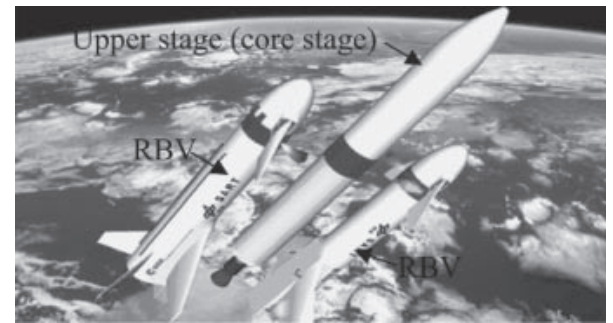

Fig. 1. The RBV and Arian 5 core stage (photo courtesy of Mattin Sippel).

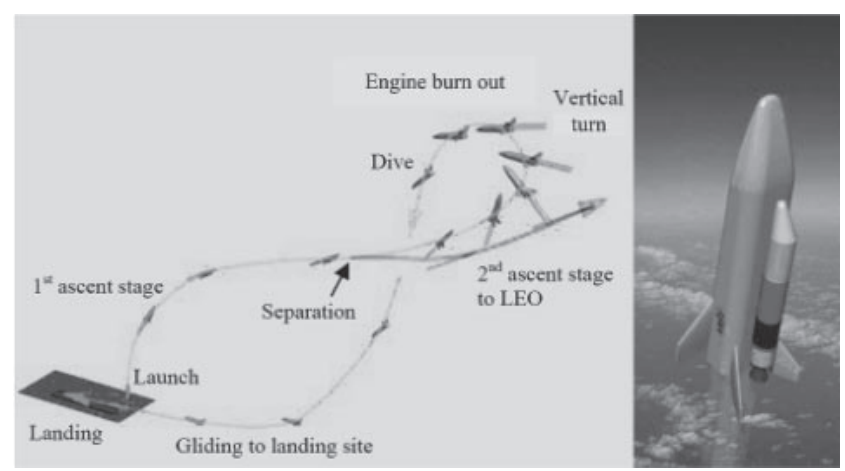

Fig. 2. The RBV and flight schematic presented by JAXA (photo courtesy of Kenji Fujii).

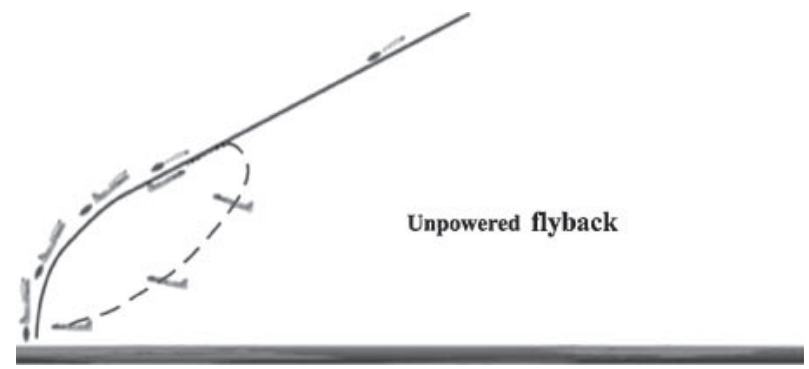

Fig. 3. Flight schematic of unpowered RBV glideback.

landing, the information from navigation systems must be credible and veracious, while providing correct datum references for the control system. Simultaneously, for the purpose of reducing cost and improving system autonomies, the flight and landing of the RBV are carried out automatically without interaction between ground facilities or people. These special application situations determine that navigation of the RBV must coincide with highly dynamic flight, and have to have a high level of accuracy, autonomy and authenticity.

For a fixed-winged and high lift-to-drag ratio booster, in order to realize automated landing safely, the booster must be sufficiently agile, requiring high bandwidth guidance and control loops; therefore, the navigation system must be updated frequently. Moreover, the limited aerodynamic capabilities of the booster, together with the strict requirements of the landing maneuvers, demand the navigation solution to be very accurate. Commonly, the attitude should updated at $50 \mathrm{~Hz}$ with $3-\sigma$ accuracies higher than $0.3^{\circ}$, and the position and velocities should updated at

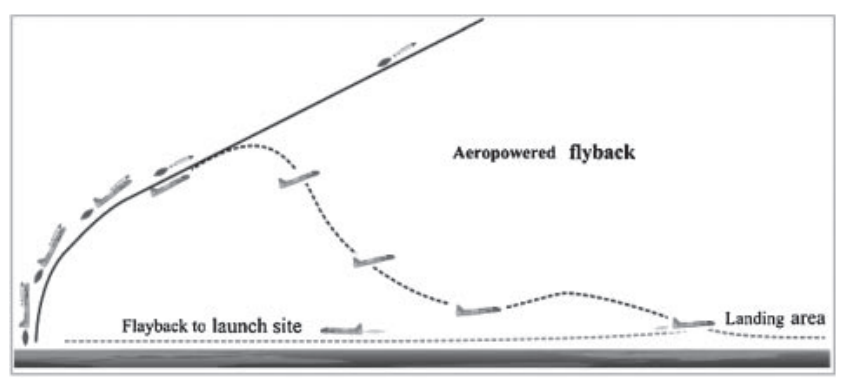

Fig. 4. Flight schematic of aero-powered RBV flyback.

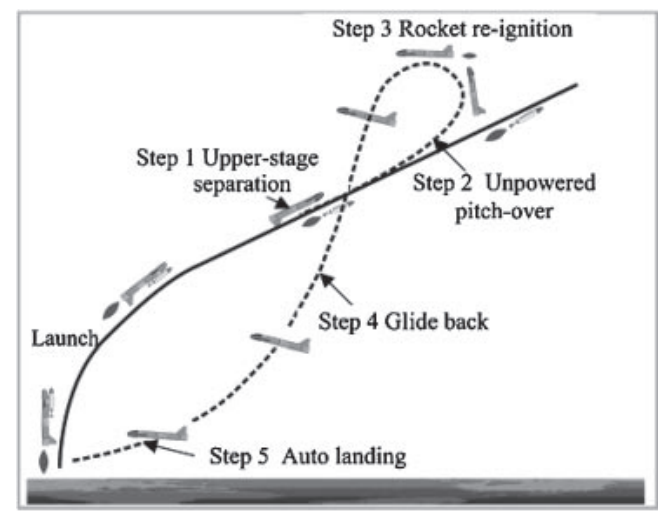

Fig. 5. Flight schematic of rocket-powered RBV flyback.

20-25 Hz with 3- $\sigma$ accuracies better than $0.5 \mathrm{~m}$ and $1.0 \mathrm{~m} / \mathrm{s}$, respectively. ${ }^{11)}$

This paper focuses on the navigation scheme and technologies for a rocket-powered flyback RBV. In contrast to other flyback options, the rocket-powered model has stricter requirements and demands higher capabilities of the navigation system. In order to carry out the pitch-over maneuver and re-ignition properly to attain a feasible reentry state, all of which are crucial for the flyback, the navigation system must be highly accurate and reliable. During the glideback and automated landing periods, the capability and efficiency of navigation determine the flight performance and whether or not the RBV can land on the runway safely.

Traditionally, a centralized navigation structure is used for spacecraft navigation, such as extended Kalman filter and federated filter. This is because the computation load increases exponentially when the length of state vector increases, especially when a fault-tolerant filter is used. Large computation loads are adverse for the fast response and dynamic adaptability of navigation systems; meanwhile, hardware requirements are more restrictive. Hence, the navigation scheme proposed here is based on dynamic information selection, and the information integration method is improved from the conventional federated filter structure. Compared with the traditional method, the navigation scheme is more dynamic and the information integration method is easier to realize, while avoiding the problems of information loss and excessive computation load during large matrix operations.

Furthermore, the selection of hardware is based on the test results of the PHOENIX experiment vehicle of the ASTRA 
program in Germany. ${ }^{11,12)}$ The aim of this study is to give an account of the navigation scheme and evaluate the improved information method through an engineering flight trajectory of rocket-powered flyback RBV. If it's necessary, other associated navigation equipment such as a laser range finder, air-data system and so on will be considered in future research.

\section{Navigation Scheme}

\subsection{Hardware configuration}

According to the flight profile, the attitude maneuvers and rocket engines' re-ignition are carried out after separation. During this time the maximum climb height and attitude control error are limited, but the position and velocity errors in the initial reentry conditions have special effects on the flight. Therefore, the integration consisting of strapdown inertial navigation system (SINS) and global navigation satellite system (GNSS) is sufficient for the navigation in this segment, and the application of additional tools such as a celestial navigation system is unnecessary.

During the first stage of reentry, the RBV keeps a constant attitude, the atmosphere density is rarefaction and the effects of the environment are neglectable. In the second stage of reentry, the RBV changes attitude to guarantee that all the restraints are observed, although the latent plasma sheath may have adverse effects on the GNSS, the navigation errors of the SINS are not serious because the impact period is limited. Furthermore, the use of radio navigation system during the following flight segment could reduce short-term navigation errors previously experienced. Therefore, the integration of SINS and GNSS will be sufficient for RBV reentry navigation.

The automated landing of the RBV has the highest requirement for navigation and control systems. Therefore, a radio navigation system comprised of Doppler radar and a radar altimeter is introduced into the application. This kind of radio navigation could improve the navigation accuracy further and ensure the realization of an accurate and reliable automated landing.

For the purpose of engineering application, the navigation system should be as simple as possible while realizing the required functions. The hardware of navigation system is comprised of the SINS, GNSS and a radio. The SINS and GNSS work during the whole flyback, but the radio system joins navigation after the RBV enters the approach and keeps working through automatic landing until touch down. The application states of the hardware during the flyback are listed in Table 1.

The SINS can provide consecutive information including position, velocity and attitude, the GNSS can provide position and velocity information, Doppler radar gives threedimensional velocities and the radar altimeter measures the altitude of the RBV from the ground. All the information from the GNSS, Doppler radar and radar altimeter could provide certain correction to the navigation errors of the SINS.
Table 1. Application states of navigation hardware.

\begin{tabular}{lcccc}
\hline Flight Stage & SINS & GNSS & Doppler & Altimeter \\
\hline Pitch-over & $\checkmark$ & $\checkmark$ & & \\
Re-ignition & $\checkmark$ & $\checkmark$ & & \\
Reentry & $\checkmark$ & $\checkmark$ & & \\
Automatic landing & $\checkmark$ & $\checkmark$ & $\checkmark$ & $\checkmark$ \\
\hline
\end{tabular}

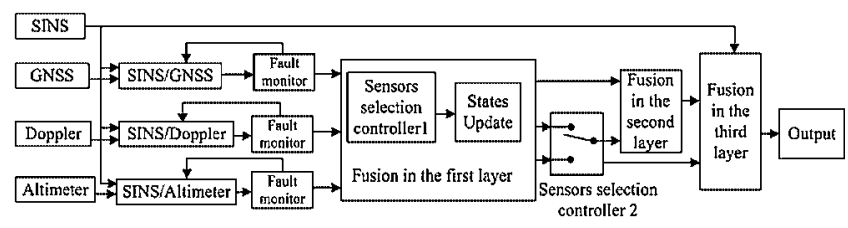

Fig. 6. Information-processing schematic.

\subsection{Information-processing strategy}

According to the highly dynamic and highly reliable application characters of RBV navigation, the informationprocessing technology must have dynamic adaptability and fault-tolerant capability. Moreover, information integration and collocation should be optimal. ${ }^{10)}$ Therefore, an active, multi-layer information-processing scheme based on dynamic Bayesian networks (DBNs) is applied to realize dynamic information fusion and global fault tolerance. ${ }^{13)}$ The whole information-processing schematic is shown in Fig. 6.

SINS/GNSS, SINS/Doppler and SINS/Altimeter are three local fault-tolerant filters in which $\chi^{2}$ fault detection is used. The federated filter is used for information fusion in all layers. The fusion results are used to correct and compensate the errors of the SINS. The sensor selection controller is used for selecting effective and additional navigation information. ${ }^{14)}$

It's well-known that the information sharing coefficient plays an important role in the federated filter and determines global fusion performance. ${ }^{15)}$ According to optimal information scheme theories in dynamic Bayesian networks, the sensor selection controller uses the information-sharing coefficient as a criterion to select and scheme navigation information. Through selecting the most effective sensor sets, the fusion method can optimize information sources dynamically and enhance the ability to correct SINS errors. In this way, the navigation precision and dynamic adaptability are apparently improved.

As shown in Fig. 6, during the approach and landing portions, the sensor selection and fusion processes are listed as follow. ${ }^{16)}$

Step 1: Based on the local filter, select the integration whose result has the largest information-sharing coefficient from SINS/GNSS, SINS/Doppler and SINS/Altimeter as the basic integration. This sensor set can reduce the errors of the SINS as much as possible during all the additional sensors at this time instant. The information of this assistant navigation system has the greatest ability of reducing the uncertainty of the integrated navigation results in the first fusion layer. 
Step 2: Take the results of the basic integration selected in Step 1 and the other two integrations as the inputs of the federated filter in the first fusion layer, and select the assistant sensor whose integration with the SINS has the largest information-sharing coefficient to extend the selected basic sensor integration.

Step 3: Based on the outputs of the selected sensor subset, run the federated filtering with the remaining sensors and calculate the information-sharing coefficients in the second fusion layer. Select the sensor whose local filter results have the largest information-sharing coefficient among the remaining sensors as the secondary effective additional sensor to extend the sensor subset and form a new sensor subset.

Step 4: Repeat Step 3, extend one sensor once into the selected sensor subset until all the useable sensors are selected and used.

Step 5: Integrate all of the information from selected sensors in the third fusion layer using the improved federated filter to get the global outputs, and use the fusion results to correct the SINS errors.

In the information-processing flow above, when one or more assistant sensors fail, the information from which is out of work partially or completely. Through the no feedback $^{16)}$ and output correct partial fusion model, ${ }^{17)}$ together with the sensor selection controller mentioned above, the spent information can be isolated clearly and the global fusion results are impregnable. If all of the assistant sensors are out of work at some instant, the sensor selection controller will choose the results of the main reference system (SINS) as the integrated navigation output. In this way, sensor faults can be isolated immediately and effectively. Simultaneously, the outputs of the normal main reference system can promote the recovery of the faulty sensors. During the process, the system transitions and reconfigurations caused by sensor selection and fault isolation are mitigated.

It is obvious that the information algorithm introduced here realizes active and dynamic sensor selection through the sensor selection controller based on the informationsharing coefficient. This method lets the system have the ability to realize fault detection isolation and recovery (FDIR ${ }^{18)}$ autonomously and timely by distinguishing the usability and validity of sensor outputs, without setting special fault-tolerant algorithms and constraints artificially.

\section{Improved Information Fusion Method}

As mentioned above, the information-sharing coefficients of a traditional federated filter are used for optimal sensor scheduling and information fusion. There are kinds of federated filter models in application, and the calculation of information-sharing coefficients can be operated using various methods. For the sake of reducing calculation load and using effective sensor information adequately to enhance the method's dynamic adaptability and fault-tolerant ability, a simple and effective method for information-sharing coefficients calculation and information fusion is proposed as follows.
Based on a comparison of different improved information fusion methods which were used commonly in recent years, a no feedback and output correct partial fusion model is used here. Under this kind of information fusion framework, the assumption that different components of the global state vector are independent is considered, so the interactive effects between them are inconsequential and negligible.

In this way, the state error covariance matrix is split into partitioned matrices. The partition matrices on the diagonal line of the state error covariance matrix reflect the main characteristics of the estimation result about the corresponding components, and different components of the global state vector are treated respectively. Therefore, the fusion results of the components comprise the global fusion results. The improved information fusion method is interpreted as follows:

There are $N$ local filters, and the global state vector is $X=[u v w]$, the output of the $i$ 'th local filter is $X_{i}=$ $\left[u_{i} v_{i} w_{i}\right]$, and the corresponding covariance matrix of the estimate error is $P_{i}=\operatorname{diag}\left(\left[\begin{array}{lll}P_{u i} & P_{v i} & P_{w i}\end{array}\right]\right)$ where $u, v$ and $w$ are the common components of local filters, which are the position, velocity and attitude or the deviation values of them in the integrated navigation system. $P_{u i}, P_{v i}$ and $P_{w i}$ are the estimate error covariance matrixes of $u, v$ and $w$ in the $i$ 'th local filter respectively. Therefore, the information-sharing coefficients for the common components are

$$
\begin{aligned}
& \beta_{u i}=P_{u i}^{-1}\left(\sum_{i=1}^{N} P_{u i}^{-1}\right)^{-1} \\
& \beta_{v i}=P_{v i}^{-1}\left(\sum_{i=1}^{N} P_{v i}^{-1}\right)^{-1} \\
& \beta_{w i}=P_{w i}^{-1}\left(\sum_{i=1}^{N} P_{w i}^{-1}\right)^{-1} .
\end{aligned}
$$

The optimal fusion results of the components are

$$
\begin{aligned}
& \hat{X}_{\mathrm{g} u}=\sum_{i=1}^{N} \beta_{u i} u_{i} \\
& \hat{X}_{\mathrm{g} v}=\sum_{i=1}^{N} \beta_{v i} v_{i} \\
& \hat{X}_{\mathrm{g} w}=\sum_{i=1}^{N} \beta_{w i} w_{i} .
\end{aligned}
$$

Hence, the global fusion result is

$$
\hat{X}_{\mathrm{g}}=\left[\begin{array}{lll}
\hat{X}_{\mathrm{g} u} & \hat{X}_{\mathrm{g} v} & \hat{X}_{\mathrm{g} w}
\end{array}\right]^{\mathrm{T}}
$$

\section{Simulation}

A simulation of RBV flyback navigation is presented here to validate the scheme and algorithms introduced in the previous section. In the simulation, gyro constant drift is 0.01 $\mathrm{deg} / \mathrm{hr}$, gyro random walk is $0.001 \mathrm{deg} / \mathrm{hr}$, accelerometer constant bias is $0.001 \mathrm{~g}$, and accelerometer random bias is $0.0001 \mathrm{~g}$. The horizontal and vertical positioning errors 


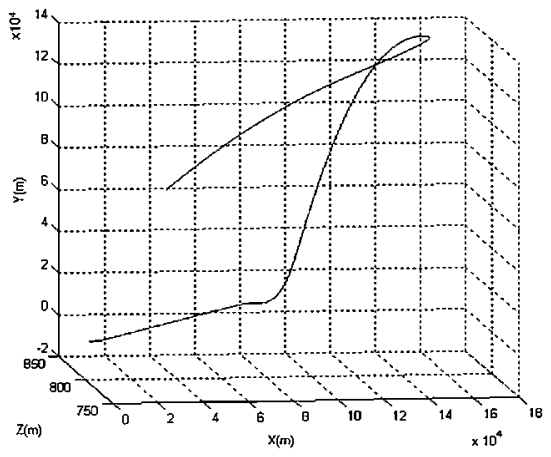

Fig. 7. 3-D flight trajectory.
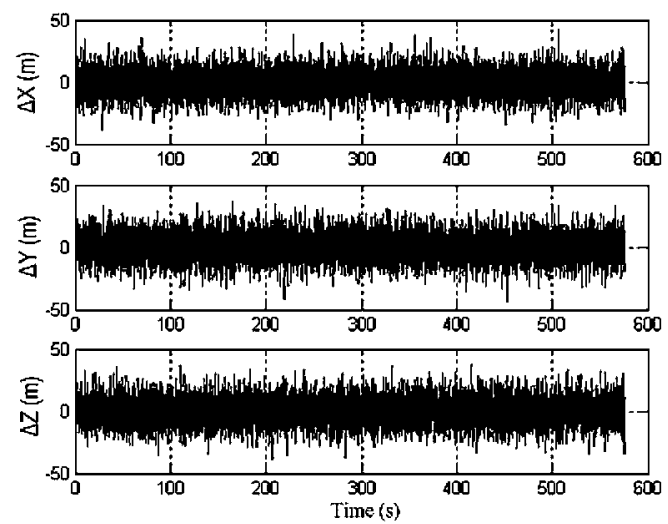

Fig. 8. Position errors of integrated navigation.
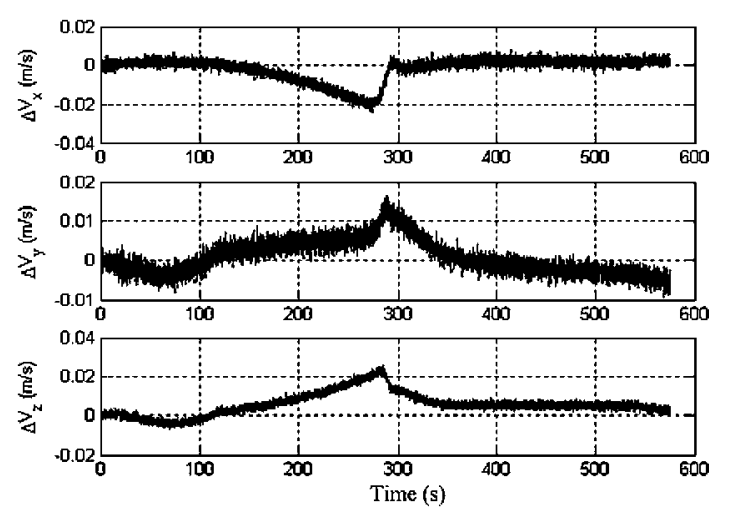

Fig. 9. Velocity errors of integrated navigation.

of the GNSS are $20 \mathrm{~m}$, and the velocity measure error is $0.5 \mathrm{~m} / \mathrm{s}$. The velocity errors of the Doppler radar are $0.05 \mathrm{~m} / \mathrm{s}$. The measurement error of the altimeter is $0.5 \mathrm{~m}$. The information refreshing period is $0.01 \mathrm{~s}$ and the fusion period is $0.1 \mathrm{~s}$.

After separation, the flight time is $580 \mathrm{~s}$ and the maximum altitude is $112.05 \mathrm{~km}$, as shown by the three-dimensional flight trajectory of the RBV in Fig. 7. At separation point, the velocity of the RBV is $1,800 \mathrm{~m} / \mathrm{s}$, the pitch angle is $40 \mathrm{deg}$ and the flight path angle is $41.5 \mathrm{deg}$.

At the same time, there are several sensor fault situations during the flight. The GNSS receiver is inoperative between $280 \mathrm{~s}$ and $300 \mathrm{~s}$ with a positioning error of $50 \mathrm{~m}$ in position and $10 \mathrm{~m} / \mathrm{s}$ in velocity. Doppler radar gets into inactivation between $300 \mathrm{~s}$ to $400 \mathrm{~s}$ with measurement outlays of $10 \mathrm{~m} / \mathrm{s}$
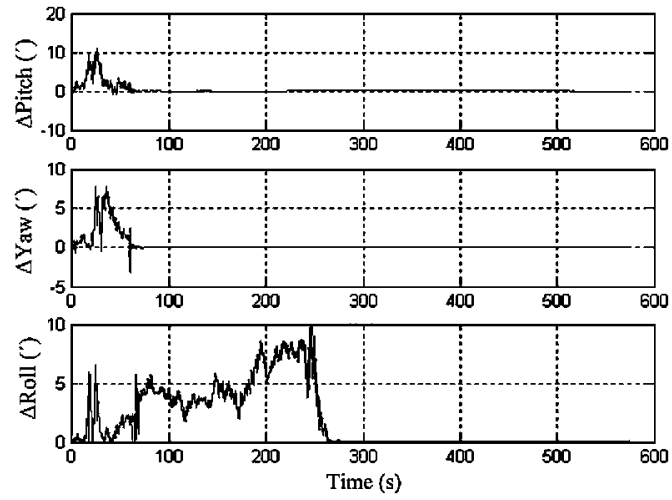

Fig. 10. Attitude errors of integrated navigation.
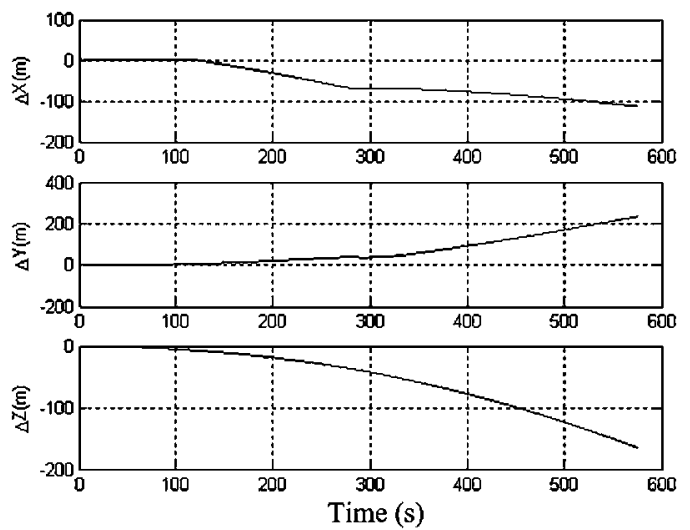

Fig. 11. Position errors of the SINS.
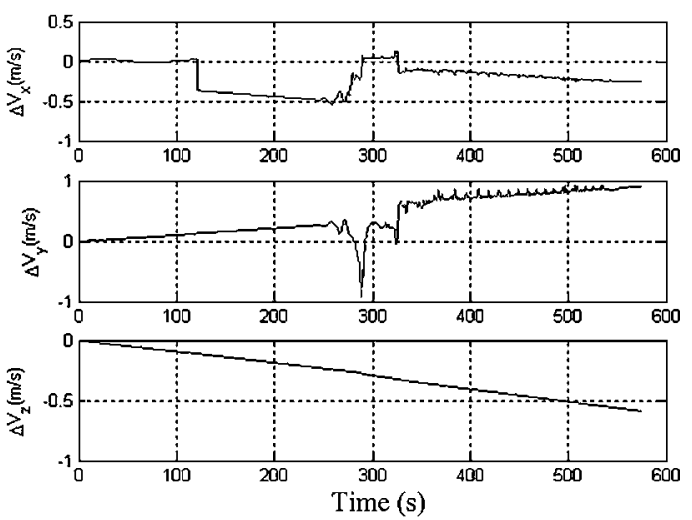

Fig. 12. Velocity errors of the SINS

in velocity. Figures $8-10$ outline the navigation results of the integrated navigation system with the algorithms proposed in this paper considering the fault situations above. The navigation errors of the RBV when using a signal SINS are shown in Figs. 11-13. The comparison of navigation results between the SINS and integrated navigation at the landing point is shown in Table 2. All of the errors mentioned above are the values for $1 \sigma$.

Simultaneously, the histories of the information-sharing coefficients for two local filters, SINS/GNSS and SINS/ Doppler/altimeter, are shown in Fig. 14 and Fig. 15. In Fig. 14 and Fig. 15, the three subplots in each figure indicate the information-sharing coefficients for attitude mis- 

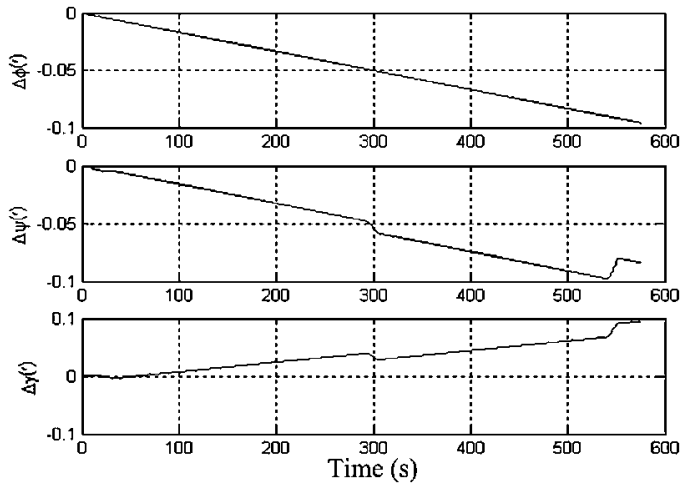

Fig. 13. Attitude errors of the SINS

Table 2. Navigation accuracy at landing point.

\begin{tabular}{llcc}
\hline & & SINS & Integrated navigation \\
\hline Position errors, $\mathrm{m}$ & $X$ & -115 & 1.5 \\
& $Y$ & 240 & 0.5 \\
& $Z$ & -165 & 2.0 \\
\hline Velocity errors, $\mathrm{m} / \mathrm{s}$ & $V_{x}$ & -0.25 & 0.002 \\
& $V_{y}$ & 0.9 & -0.008 \\
& $V_{z}$ & -0.6 & 0.005 \\
\hline \multirow{2}{*}{ Attitude errors, $\left(^{\prime}\right)$} & Pitch & -0.1 & 0.02 \\
& Yaw & -0.08 & 0.01 \\
& Roll & 0.1 & 0.02 \\
\hline
\end{tabular}

alignment angle, position, and velocity in three directions, respectively, from top to bottom.

The results in Fig. 14 and Fig. 15 are zoomed in to show the change histories of the information-sharing coefficients. The phenomenon that the changes of $\beta$ do not correspond to the sensor fault scenarios is attributed to two reasons. The first is that the velocity error of the SINS/Doppler/altimeter is even lower than the SINS/GNSS when the Doppler radar is out of work, and the position error of the SINS/GNSS is much lower than the SINS/Doppler/altimeter during the periods when the GNSS is disturbed or inoperative. The second is caused by information loss because we split the covariance matrix into partitioned matrices and only consider the matrices on the diagonal line, there may be some information loss inevitably. Although this method could obtain satisfactory navigation results, the information-sharing coefficients couldn't completely and strictly represent the changes in sensor information. The detailed descriptions to technically justify this method will be introduced in our next paper in the near future.

Apparently, the integrated navigation system stops navigation errors from accumulating over time, and effectively improved the navigation accuracy. At first glance, it seems that the positioning accuracy is not sufficiently high, but the positioning errors are less than the GNSS errors throughout almost all of the flight period. The jump in errors in Fig. 9 is mainly the result of the random errors of the GNSS. By closely reviewing the results, it is obvious that the dynamic information fusion model and algorithm proposed
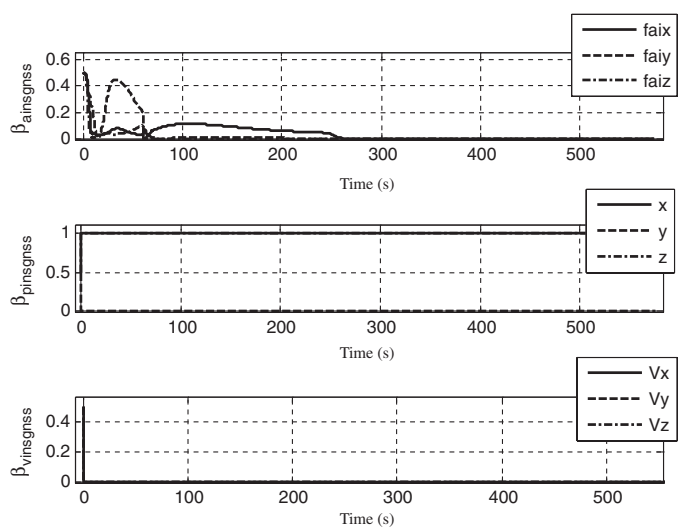

Fig. 14. Information-sharing coefficients of SINS/GNSS.
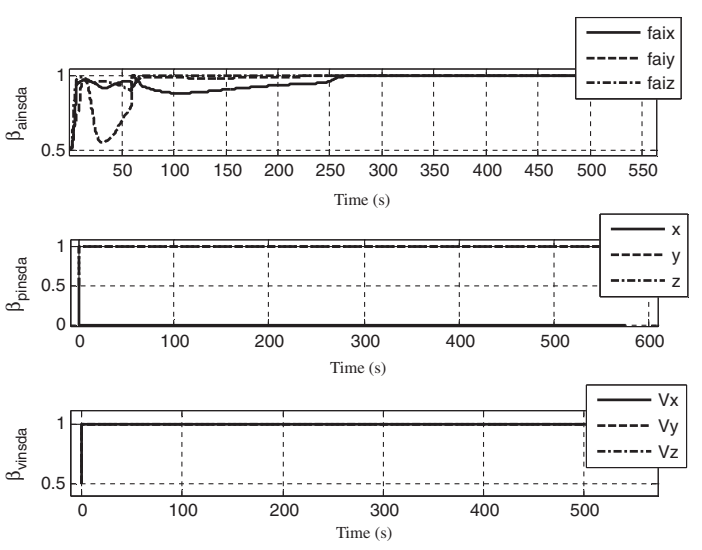

Fig. 15. Information-sharing coefficients of the SINS/Doppler/altimeter.

here can isolate the sensor faults and improve the navigation accuracy effectively: the positioning error is no more than $2 \mathrm{~m}$, the velocity accuracy is higher than $0.1 \mathrm{~m} / \mathrm{s}$ and the attitude error is a maximum of $0.02 \mathrm{~min}$ at the landing point.

Compared with traditional methods in which a signal SINS is used for navigation, the navigation system and information fusion approach presented here is more effective and feasible.

\section{Conclusion}

This paper introduced the development states and three typically used flyback options of RBVs. Taking the rocketpowered flyback RBV as an example, the navigation scheme, hardware components and information-processing flow are proposed. This paper improved the traditional federated filter and proposed a dynamic information fusion model and algorithm for multi-sensor systems based on DBNs theories. The method presented here is simple to apply and can realize optimal dynamic scheduling autonomously. The simulation applying the algorithm for the RBV flyback navigation system validated the great dynamic adaptability and fault-tolerant ability of the model and algorithm.

In contrast to the traditional federated filter, the improved fusion method avoids the problem of inverse operation to a 
large matrix and the shortcomings of information loss during many excessively simplified methods. However, the calculation load is minimal, so the fault tolerance and dynamic adaptability are high. From the point of application, the navigation system can be used for sub-orbit vehicles, hypersonic spacecraft and so on. Based on the refined analysis and theoretical demonstration, even further improvements in the system will be the focus of future work.

\section{References}

1) Deneu, F., Malassigne, M., Le-couls, O. and Baiocco, P.: Promising Solutions for Fully Reusable Two-stage-to-orbit Configurations, Acta Astronautica, 56 (2005), pp. 729-736.

2) Sippel, M., Manfletti, C. and Burkhardt, H.: Long-term/Strategic Scenario for Reusable Booster Stages, Acta Astronautica, 58 (2006), pp. 209-221.

3) Stone, D. and Lindenmoyer, A.: NASA's Approach to Commercial Cargo and Crew Transportation, Acta Astronautica, 63 (2008), pp. 192-197.

4) Minami, Y., Ishimoto, S., Mori, T. and Fujii, K.: Design Study on a Small-Sized Partially Reusable Launch System, AIAA/CIRA 13th International Space Planes and Hypersonics Systems and Technology Conference, AIAA, Capua, Italy, 2005, AIAA 2005-3249, pp. 1-12.

5) Fujii, K. and Ishimoto, S.: Research Activities to Realize Advanced Space Transportation System, 15th AIAA International Space Planes and Hypersonics Systems and Technologies Conference, Dayton Ohio, 2008, AIAA, AIAA 2008-2575, pp. 1-7.

6) Eymar, P. and Obersteiner, M.: A Semi-expendable Vehicle as RLV Precurso, Acta Astronautica, 55 (2004), pp. 611-618.

7) Klevanski, J. and Sippel, M.: Special Aspects of Flight Dynamics of a Reusable Cryogenic Booster Stage, Proceedings of the fifth European Symposium on Aerothermodynamics for Space Vehicles, Cologne, Germany, 2004, pp. 49-54.

8) Pamadi, B. N., Tartabini, P. V., Starr, B. R.: Ascent, Stage Separation and Glideback Performance of a Partially Reusable Small Launch
Vehicle, 42nd AIAA Aerospace Science Meeting and Exhibit 5-8 January 2004, Reno, Nevada, AIAA 2004-876, pp. 1-16.

9) Crocker, A. M, and Andrews, D. G.: Go Horizontal: A Responsible, Evolvable, Feasible Space Launch Roadmap, Space Conference and Exhibit 28-30 September 2004, San Diego, California, AIAA, AIAA 2004-6004, pp. 1-7.

10) Zhang, Y. and Ji, Q.: Active and Dynamic Information Fusion for Multisensor Systems with Dynamic Bayesian Networks, IEEE Trans. Syst. Man Cybernetics B Cybernetics, 36 (2006), pp. 467-472.

11) Voegt, S., Tava, M. and Schlotterer, M.: The PHOENIX navigatioin system for automatic landing. 10th Saint Petersburg International Conference on Integrated Navigation Systems, Saint Peterburg, Russia, May, 2003, pp. 1-8.

12) Sotto, E. D., Branco, J., Savino, R., Stefano, F. De., Monti, R., Janovsky, R., Scheper, M., Apeldoorn, J. and Molina, R. C.: PHOEBUS: GNC Design and Performance Assessment for Super Orbital Re-entry, 16th AIAA/DLR/DGLR International Space Planes and Hypersonic Systems and technologies Conference, AIAA, Bremen, Germany, 2009, AIAA 2009-7382, pp. 1-13.

13) Murphy, K. P.: Dynamic Bayesian Networks: Representation, Inference and Learning, Ph.D. Dissertation, Computer Science Dept., Univ. of California, Berkeley, 2002, pp. 35-40.

14) Zhang, Y.: Active and Dynamic Information Fusion with Bayesian Networks, Ph.D. Dissertation, University of Nevada, Reno, 2004, pp. 58-75.

15) Ali, J. and Jiancheng, F.: SINS/ANS/GPS Integration using Federated Kalman Filter Based on Optimized Information-Sharing Coefficients, AIAA Guidance Navigation and Control Conference and Exhibit, San Francisco, California, 2005, AIAA 2005-6452, pp. 6028-6039.

16) Han, P., Mu, R. and Cui, N.: Active and Dynamic Multi-sensor Information Fusion Method Based on Dynamic Bayesian Networks, 2009 IEEE International Conference on Mechatronics and Automation, IEEE, Changchun, Jilin, China, 2009, pp. 3076-3080.

17) Naigang, C., Pengxin, H. and Rongjun, M.: SINS/GPS/CNS Integrated Navigation Method Based on Microminiaturized Sensors, J. Chin. Inertial Technol., 16 (2008), pp. 68-72.

18) Da, R. and Lin, C. F.: Failure Detection and Isolation Structure for Global Positioning System Autonomous Integrity Monitoring, J. Guid., Control Dynam., 18 (1995), pp. 291-297. 